\title{
Postoperative Adjuvant Chemotherapy Cancel Out the Negative Survival Impact on Stage II/III Gastric Cancer Patients With Postoperative Complications
}

\section{Li-li Shen}

Fujian Medical University Union Hospital

Jun Lu

Fujian Medical University Union Hospital

Jia Lin

Fujian Medical University Union Hospital

Bin-bin Xu

Fujian Medical University Union Hospital

\section{Zhen Xue}

Fujian Medical University Union Hospital

\section{Dong Wu}

Fujian Medical University Union Hospital

Hua-long Zheng

Fujian Medical University Union Hospital

Guo-sheng Lin

Fujian Medical University Union Hospital

Jiao-bao Huang

Fujian Medical University Union Hospital

Jian-wei Xie

Fujian Medical University Union Hospital

\section{Ping Li}

Fujian Medical University Union Hospital

\section{Chang-ming Huang}

Fujian Medical University Union Hospital

Chao-hui Zheng ( $\nabla$ wwkzch@163.com )

Fujian Medical University Union Hospital

\section{Research Article}

Keywords: 
Posted Date: January 13th, 2022

DOI: https://doi.org/10.21203/rs.3.rs-1228368/v1

License: (c) (i) This work is licensed under a Creative Commons Attribution 4.0 International License. Read Full License 


\section{Abstract}

Purpose The potential additive influence of adjuvant chemotherapy (AC) on prognosis of patients with stage II/III gastric cancer (GC) who experienced complications after radical surgery is unclear.

Methods The whole group was divided into a postoperative complication (PC) group and a postoperative non-complication (NPC) group, and the overall survival (OS) rate, recurrence-free survival (RFS) rate and recurrence rate were compared between the two groups of patients.

Results A total of 1563 patients between January 2010 and December 2015 in our center were included in this analysis. There were 268 patients (17.14\%) in the PC group and 1295 patients (82.86\%) in the NPC group. The 5-year OS rate of the PC group was $55.2 \%$, the NPC group was $63.3 \%$; and the 5 -year RFS rate of the PC group was $53.7 \%$, the non-PC group was $58.8 \%$. Recurrence patterns showed no significant difference between the two group (all $p>0.05$ ). Adjuvant chemotherapy (AC) significantly improved the OS and RFS rates of patients with and without PCs (both $p<0.05$ ), and it showed no significant difference between the PC group and the NPC group who received AC (both $p>0.05$ ). Stratified analysis showed that AC only improve the OS or RFS rates of stage III patients (both $p<0.05$ ). Further stratified analysis of the time interval (TI) from operation to initiation of AC in the PC group showed that a TI after 6 weeks ( $\geq 6$ eeks) improved only the OS and RFS rates of stage III patients, while when a TI within 6 weeks ( $\mathbb{}$ 6 weeks), a benefit was observed in stage II and III patients (both $p<0.05$ ).

Conclusion AC can abolish the negative effect of PCs on the long-term survival of patients with stage III GC; for stage II patients, the above offset effect is affected by the TI. Delaying AC initiation after 6 weeks may not improve the survival of patients experienced stage II GC with complications.

\section{Introduction}

Gastric cancer (GC) is the fifth most common malignant tumor in humans and ranks third in tumorrelated mortality [1]. Surgery is the main treatment for GC, but the incidence of complications after radical gastrectomy plus D2 lymph node dissection remains high, at approximately $15-50 \%$ [2-5]. Previous literature has shown that postoperative complications significantly reduce the overall survival rate of patients with malignant tumors and are closely related to the increased risk of disease recurrence [6-12]. Scholars such as Sierzega M, Bohle B and others believe that complications prolong the postoperative inflammation time of patients and then aggravate inflammatory factors to stimulate the proliferation and growth of circulating cancer cells, which may lead to a poor long-term prognosis of patients after surgery $[10,13]$.

In East Asia, adjuvant chemotherapy (AC) for patients with stage II/III GC after D2 radical resection has become the standard treatment plan [11-14]. However, the occurrence of postoperative complications may prolong the time to chemotherapy initiation for patients with GC, and whether the delayed initiation of AC could affect the survival outcome of these patients remains unclear. In recent years, studies have shown that although some patients experience complications after colorectal cancer resection, the 
initiation of AC treatment "as soon as possible" reduces the increased risk of tumor recurrence in these patients due to complications and improves overall survival $[15,16]$. However, related studies in patients with GC have not been reported. This study aimed to evaluate whether AC can abolish the poor prognosis of patients who undergo radical GC surgery caused by PCs and whether the time interval(TI)from radical operation to initiation of postoperative adjuvant chemotherapy for gastric cancer patients has an impact on this abolishment. The aim of this study was to explore the effect of AC on the prognosis of GC patients with postoperative complications.

\section{Materials And Methods}

\section{Patients}

The clinicopathological data of 2476 patients who underwent radical GC surgery between January 2010 and December 2015 in the Department of Gastric Surgery, Union Hospital of Fujian Medical University, were retrospectively analyzed. The exclusion criteria were as follows: 1 . Age $<20$ years or $>80$ years $(n=67) ; 2$. Neoadjuvant chemotherapy $(n=63) ; 3$. Remnant stomach cancer $(n=59) ; 4$. The clinicopathological data were not complete $(n=36)$; and 5. pStage I $(n=688)$. Ultimately, a total of 1563 patients were enrolled (Table 1), of whom 268 were in the postoperative complication (PC) group, and 1295 were in the postoperative non-complication (NPC) group (see the flow chart in supplement material eFig. 1 for details). And our patients never experienced radiotherapy. Both preoperative clinical staging and postoperative pathological staging were carried out according to the 8th edition of the AJCC-TNM staging system [17]. All operations were performed by the same experienced surgical team. All patients signed an informed consent form. This study was approved by the FMUUH Ethics Committee.

\section{Statistical Analysis}

Continuous variables are reported as the means \pm SDs. Categorical and continuous variables were compared using a $\chi 2$ test or Fisher's exact test and a t test, respectively. The cumulative survival rate was calculated using the Kaplan-Meier method and a log-rank test. A Cox proportional hazards regression model was used to determine the independent prognostic factors associated with OS and RFS. Variables with a $p$ value $<0.05$ in the univariate analysis were subsequently entered into the multivariate Cox proportional hazards analysis. All statistical analyses were performed using SPSS v.26.0 for Windows (SPSS Inc., Chicago, IL, USA). P values less than 0.05 were considered statistically significant.

\section{Definitions}

Postoperative complication (PC) group: According to previous studies, we recorded all complications that occurred in all patients within one month after surgery and used the CD classification [18] to classify the severity of postoperative complications. Patients with grade II and above complications ( $\geq$ grade II CD) were defined as the PC group. Patients without complications and complication events below grade II were defined as the NPC group [19]. 
Adjuvant chemotherapy (AC): Adjuvant chemotherapy was recommended for patients with pathological stages II and III[20]. The patient has received at least one cycle of postoperative adjuvant chemotherapy. Based on 5-fluorouracil treatment, Most patients received a chemotherapy regimen based on S-1 plus platinum $49.5 \%$. Those who never received any adjuvant chemotherapy were defined as nonchemotherapy (NAC) patients.

The time interval (TI)from radical operation to initiation of postoperative adjuvant chemotherapy for gastric cancer patients was defined as the time from the date of surgery to the initiation of postoperative adjuvant chemotherapy. According to tumor-related diagnoses and treatment guidelines, the first postoperative chemotherapy time for GC patients is recommended to be within 6 weeks [21]. Therefore, in this study, 6 weeks was used as the observation cutoff point. Patients were divided into a TI within 6 weeks (<6eeks) group, a TI after 6 weeks ( $\geq 6$ weeks) group and a never received postoperative adjuvant chemotherapy (NAC) group.

Overall survival (OS): OS was defined as the time from the date of surgery to death or the last follow-up. All patients were followed up until death or at least 60 months, with a median follow-up time of 60 months (4-124 months).

Recurrence: Recurrence was confirmed by an imaging examination or biopsy of suspicious lesions. Recurrence-free survival (RFS) was defined as the period from the first day after surgery to the discovery of recurrence. For RFS, if the deceased patient did not have tumor recurrence, the last follow-up record was reviewed. The recurrence patterns were classified as local recurrence (LR) (anastomotic or remnant stomach), peritoneal metastasis (PM) (including positive ascites in tumor cells, progressive thickening of the peritoneum found on imaging, newly emerging peritoneal lymphadenopathy, or tumor invasion into the female uterus or ovaries) and distant metastasis (DM) (including lymph nodes outside the D2 range and liver, lung, bone and other organs) [22].

\section{Results}

\section{Clinicopathological data}

Table 1 shows a comparison of the general clinicopathological data of the PC group (268 patients) and the NPC group (1295 patients). Compared with the NPC group, the PC group had older patients, a longer operation time and more blood loss during the operation $(p<0.05)$. However, there was no significant difference in pathological staging between the two groups $(p>0.05)$.

Table 1. Clinicopathological characteristics of postoperative complications(PC) group and noncomplications(NPC) group. Note: ASA: American Society of Anesthesiologists 


\begin{tabular}{|c|c|c|c|}
\hline Variables & $\begin{array}{l}\text { PC group } \\
(n=268)\end{array}$ & $\begin{array}{l}\text { NPC group } \\
(n=1295)\end{array}$ & $P$ values \\
\hline Gender n (\%) & & & .188 \\
\hline Male & $210 \rrbracket 78.4 \% \bigotimes$ & $962(74.3 \%)$ & \\
\hline Female & $58 \otimes 21.6 \% \bigotimes$ & $333(25.7 \%)$ & \\
\hline Age(mean $\pm S D$, years $)$ & $63.14 \pm 9.79$ & $60.25 \pm 10.72$ & .000 \\
\hline ASA & & & .077 \\
\hline$\varangle 3$ & $253 \rrbracket 94.4 \% \bigotimes$ & $1252 \rrbracket 96.7 \% \rrbracket$ & \\
\hline$\geq 3$ & $15 \rrbracket 5.6 \% \rrbracket$ & $43 \rrbracket 3.3 \% \rrbracket$ & \\
\hline Tumor diameter & & & .139 \\
\hline$\nabla 50$ & $113 \llbracket 42.2 \% \rrbracket$ & $611 \llbracket 47.2 \% \rrbracket$ & \\
\hline$\geq 50$ & $155 \llbracket 57.8 \% \rrbracket$ & $684 \llbracket 52.8 \% \rrbracket$ & \\
\hline Tumor location & & & .951 \\
\hline Upper & $71 \otimes 26.5 \% \bigotimes$ & $333 \llbracket 25.7 \% \rrbracket$ & \\
\hline Middle & $58 \rrbracket 21.6 \% \bigotimes$ & $279 \llbracket 21.5 \% \rrbracket$ & \\
\hline Lower & $99 \llbracket 36.9 \% \rrbracket$ & $501(38.7 \% \bigotimes$ & \\
\hline Mix & $40 \otimes 14.9 \% \bigotimes$ & $182 \bigotimes 14.1 \% \bigotimes$ & \\
\hline BMI(mean $\pm S D, k g / m 2)$ & $22.57 \pm 3.15$ & $22.17 \pm 5.63$ & .272 \\
\hline Blood lost median(rang) ML & $135.76 \pm 266.35$ & $84.89 \pm 120.27$ & .000 \\
\hline Surgical time median(rang) min & $201.76 \pm 68.49$ & $186.39 \pm 57.41$ & .000 \\
\hline Type of surgery & & & .090 \\
\hline Total & 169₫63.1\%】 & 767(ه59.2\%区 & \\
\hline Distal & $94 \rrbracket 35.1 \% \rrbracket$ & $518 \rrbracket 40.0 \% \rrbracket$ & \\
\hline Proximal & $5 \rrbracket 1.9 \% \rrbracket$ & $10 \otimes 0.8 \% \rrbracket$ & \\
\hline Vessel/ Lymphatic infiltration & & & .241 \\
\hline Present & 103『38.4\%》 & $449 \llbracket 34.7 \% \rrbracket$ & \\
\hline Absent & $165 \rrbracket 61.6 \% \rrbracket$ & $846 \rrbracket 65.3 \% \rrbracket$ & \\
\hline Nerve infiltration & & & .020 \\
\hline Present & $98 \rrbracket 36.6 \% \rrbracket$ & $378 \rrbracket 29.2 \% \rrbracket$ & \\
\hline
\end{tabular}




\begin{tabular}{|c|c|c|c|}
\hline Absent & $170 \rrbracket 63.4 \% \rrbracket$ & $917 ه 70.8 \% \rrbracket$ & \\
\hline pT stage & & & .164 \\
\hline 1 & $1 \rrbracket 0.4 \% \rrbracket$ & $32 \rrbracket 2.5 \% \rrbracket$ & \\
\hline 2 & $21 \otimes 7.8 \% \bigotimes$ & $113 \rrbracket 8.7 \% \rrbracket$ & \\
\hline 3 & $123 \rrbracket 45.9 \% \bigotimes$ & $585 \rrbracket 45.2 \% \bigotimes$ & \\
\hline 4 & $123 \rrbracket 45.9 \% \rrbracket$ & $565 \rrbracket 43.6 \% \rrbracket$ & \\
\hline pN stage & & & .925 \\
\hline NO & $41 \otimes 15.3 \% \bigotimes$ & 195囚15.1\%区 & \\
\hline $\mathrm{N} 1$ & $227 \llbracket 84.7 \% \rrbracket$ & $1100 \rrbracket 84.9 \% \rrbracket$ & \\
\hline pTNM stage & & & .669 \\
\hline प & $85 \llbracket 31.7 \% \rrbracket$ & $431 \otimes 35.1 \% \bigotimes$ & \\
\hline$\square$ & $183 \rrbracket 68.3 \% \rrbracket$ & $864 \llbracket 66.7 \% \rrbracket$ & \\
\hline Adjuvant chemotherapy & & & .022 \\
\hline Yes & $196 \rrbracket 73.1 \% \bigotimes$ & $1031 \rrbracket 79.6 \% \rrbracket$ & \\
\hline No & $72 \rrbracket 21.4 \% \rrbracket$ & $264 \rrbracket 20.4 \% \rrbracket$ & \\
\hline Type of adjuvant chemotherapy & & & 0.073 \\
\hline Platinum & $110 \otimes 56.1 \% \bigotimes$ & $497 \rrbracket 48.2 \% \rrbracket$ & \\
\hline Capecitabine & $48 \rrbracket 24.5 \% \rrbracket$ & $334 \llbracket 32.4 \% \rrbracket$ & \\
\hline Paclitaxel & $13 \rrbracket 6.6 \% \bigotimes$ & $49 ه 4.8 \% \rrbracket$ & \\
\hline Others & $25 \bigotimes 12.8 \% \rrbracket$ & $151 \otimes 14.6 \% \rrbracket$ & \\
\hline
\end{tabular}

\section{Surgical morbidity and mortality}

The details of the complications in all patients are shown in eTable.1 (Supplement material eTable.1). Pulmonary infection was the most frequent morbidity ( 89 cases, $6.78 \%$ ), followed by Intra-abdominal infection (60 cases, 3.83\%) and anastomotic leakage (34 cases, $2.17 \%$ ). Wound complications are the rarest (16 cases, $1.08 \%)$. Complications of grade II were found in 206 cases (13.18\%), grade III in 34 cases $(2.18 \%)$, while those of grade IV or $\mathrm{V}$ occurred in 28 cases $(1.8 \%)$. Surgical mortality was never observed.

\section{Survival analysis}


As shown in Fig. 2, the 5-year OS rate of the PC group was significantly lower than that of the NPC group (55.2\% vs 63.3\%, $\mathrm{p}=0.016)$; the 5-year RFS rate of the PC group was lower than that of the NPC group, but the difference was not statistically significant $(53.7 \%$ vs $58.8 \%, p=0.14)$. A univariate analysis of the entire population, PC group and NPC group showed that age, tumor size, tumor location, vascular lymphatic infiltration, nerve infiltration, and adjuvant chemotherapy were closely related to OS and RFS at 5 years after surgery (all $p<0.05$, Supplement material eTable 5 , eTable 7 ). Multivariate analysis showed that age, tumor size, tumor location, vascular lymphatic invasion, nerve invasion, and postoperative adjuvant chemotherapy were independent prognostic factors for 5-year OS and RFS in the entire population and NPC group (all p<0.05), and tumor size [OS: HR=2.062 (1.362-3.122), $p<0.000$; RFS: $H R=1.968$ (1.319-2.936), $p<0.000$ ], nerve infiltration [OS: $H R=2.236$ (1.575-3.176), $p<0.000$; RFS: $H R=1.193$ (1.312-2.791), $p=0.001$ ] and adjuvant chemotherapy [OS: HR=0.498 (0.316-0.783), $p=0.003$; RFS: HR=0.485 (0320-0.737), $p=0.001]$ were identified as independent prognostic factors of OS and RFS in patients with PCs at 5 years after the operation (Supplement material eTable 6, eTable 8).

\section{Recurrence patterns}

Fig. 1A shows that there were 124 (46.3\%) and 533 (41.2\%)recurrences in the PC group and NPC group, respectively. In the PC group, 11 patients had local recurrence (4.1\%), 40 had peritoneal metastasis (13.8\%), and 87 had distant metastasis (32.5\%); in the NPC group, 70 patients (5.4\%) had local recurrence, 160 (12.4\%) had peritoneal metastasis, and 380 (29.3\%) had distant metastasis; The median times to recurrence in the two groups were 17.6 months and 15.8 months. in the PC group, the median time to local recurrence was 55 months, that to peritoneal metastasis was 24 months, and that to distant metastasis was 14.7 months; in the NPC group, the median time to local recurrence was 50 months, that to peritoneal metastasis was 17.7 months, and that to distant metastasis was 16.6 months; There was no significant difference in the location of recurrence or time to recurrence between the two groups ( $p>0.05$ ) (Supplement material eTable 2). According to the stratified analysis, there was no significant difference in the location of recurrence or time to recurrence between stage II and stage III patients (p>0.05) (Fig. 1B, Fig. 1C; Supplement material eTable 3, eTable 4).

\section{The effect of adjuvant chemotherapy (AC)}

According to whether adjuvant chemotherapy (AC) was received, a stratified analysis of the PC group and NPC group showed that AC significantly improved the 5-year OS and RFS rates of patients with and without PCs (both $\mathrm{p}<0.05$ ). In addition, the 5-year OS and RFS rates of patients in the PC group who received $A C$ were significantly higher than those of patients without adjuvant chemotherapy(NAC) group (both $p<0.05$ ), and there was no significant difference in the 5-year OS and RFS rates between the PC group and NPC group when they were given AC. (both $p>0.05$ ) (Fig. 3A, Fig. 3B).

Further stratified analysis based on pathological staging showed that in stage II patients with PCs (Fig. 3C, Fig. 3D), AC did not significantly improve 5-year OS or RFS (both $p>0.05$ ). In stage III patients (Fig. 3E, Fig. 3F), the 5-year OS and RFS rates of the PC group who received AC were significantly higher than NAC group (both $p<0.05$ ). 


\section{The effect of the time interval (TI) from radical operation to initiation of adjuvant chemotherapy}

eFig. 2A(Supplement material eFig. 2A) shows the distribution of the TI. The median TI of patients with PCs was 36 days. The 5-year OS and RFS rates of patients in the TI $\geq 6$ weeks group and TIX 6 weeks group were significantly better than those of patients in the NAC group (both $p<0.05$ ) (Fig. 3G, Fig. 3H).

The stratified analysis of pathological stage showed that the median TI of stage II and stage III patients with PCs were 35 days and 36 days, respectively (Supplement material eFig. 2B, eFig. 2C). Among the stage II patients, only the 5 -year OS $(p=0.038)$ and RFS $(p=0.005)$ rates of patients with PCs in the TI\&6 weeks group were significantly higher than NAC group, while a TI $\geq 6$ weeks did not significantly improve the 5-year OS and RFS rates of stage II PC group (Fig. 3I, Fig. 3J). Among stage III patients, the 5-year OS

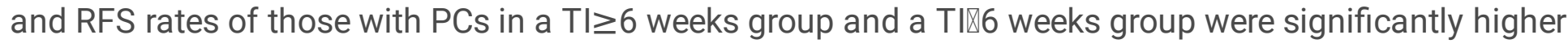
than NAC group (both $p<0.05$ ) (Fig. 3K, Fig. 3L).

\section{Discussion}

In recent years, an increasing number of scholars have reported that postoperative complications (PCs) after surgical operations are closely related to the poor prognosis of patients with malignant tumors [2327]. This retrospective study analyzed this adverse effect and explored whether adjuvant chemotherapy (AC) and the time interval ( $\mathrm{TI}$ ) from radical operation to initiation of adjuvant chemotherapy could abolish the poor prognosis caused by PCs in patients with stage II/III GC. We found that compared with the NPC group, the long-term survival rate of the PC group was lower; AC significantly improved the OS and RFS rates of the NPC group and the PC group. Further stratified analysis of the $\mathrm{TI}$ in patients with PCs showed that in stage III patients with PCs, a TI $₫ 6$ weeks and a TI $\geq 6$ weeks could reduce the risk of disease recurrence and improve overall survival; however, in PC-II patients, only a TI囚6 weeks could significantly improve 5 -year OS $(p=0.038)$ and RFS $(p=0.005)$.

At present, surgical resection remains the primary method for the treatment of GC, but the occurrence of complications after gastrectomy cannot be completely avoided. Previous reports on the mechanism of postoperative complications negatively affecting the prognosis of patients with malignant tumors have been inconsistent. JH Saunders et al. found that postoperative anastomotic leakage did not affect the long-term survival of patients with esophageal cancer, but other serious complications (except for anastomotic leakage) were identified as independent prognostic factors for overall survival and diseasefree survival [25]. In the field of GC, Masanori et al. found in the JCOG1001 study that, regardless of the type or degree of complications after radical GC, postoperative complications were associated with poor OS [26]. Kubota et al. analyzed 1395 patients with GC who underwent radical resection and found that compared with patients without complications, the 5-year survival rate of patients with postoperative complications was significantly lower, and they believed that this finding may have been related to complications. Complications are related to a prolonged postoperative inflammation time [12]. In addition, studies by Katai $\mathrm{H}, \mathrm{Okholm} \mathrm{C}$ and others [28-30] have shown that postoperative complications may actually alter the progression of the malignant tumor itself. Inflammatory mediators and cellular 
effects are important parts of the local tumor environment. PC-induced systemic inflammatory responses through inflammatory mediators[31,32], such as interleukin-6[33,34], nuclear factor-kappa B[35] and Creactive protein[36], stimulate residual microbes after surgery and the proliferation of metastatic tumor cells.

$A C$ is usually administered to remove residual cancer cells and micro-metastasis after surgery, and it has been proven that AC after GC resection can benefit patient survival [37-39]. However, there is no conclusive conclusion about the time at which patients with PCs should start postoperative adjuvant chemotherapy. In recent studies on PCs in patients undergoing GC resection, it has been shown that patients with PCs and delayed AC initiation have reduced survival rates [39-42]. It has also been reported that the delayed initiation of $\mathrm{AC}$ is associated with poor survival outcomes for patients with colorectal cancer, ovarian cancer, and breast cancer [43-45]. In the field of GC, a meta-analysis of 6017 patients showed that every 4-week delay in postoperative adjuvant chemotherapy initiation reduced the relative OS rate by $14 \%$ [46]. In this study, we found that in stage II patients with PCs, when the TI was advanced to less than 6 weeks, the 5-year OS and RFS rates were significantly improved. For stage III patients with PCs, chemotherapy can be started within 6 weeks or after 6 weeks and significantly improve 5-year OS and RFS. This may be because inflammatory mediators and cell effect factors are an important part of the local tumor environment, and the inflammatory state caused by PCs increases the risk of blood recurrence in advanced GC [47], which is related to poor long-term survival. The tumor burden of stage II patients is low, and the number of circulating cancer cells may be small. If the body can tolerate the

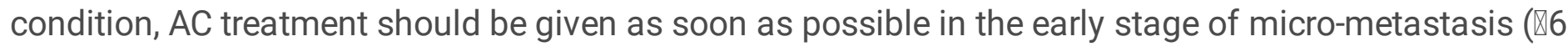
weeks), as it helps to inhibit tumor cell proliferation, reduce the risk of recurrence and improve overall survival.

This study has the following shortcomings: 1 . This was a single-center retrospective study that lacked external verification, and the sample size was small. A large-sample multicenter study is needed to further confirm the conclusions of this study. 2. In this study, we did not evaluate whether the course of chemotherapy affects the long-term prognosis of patients. 3. The effect of chemotherapy on prognosis was not studied. Nevertheless, to the best of our knowledge, this study was the first to investigate the effect of AC on the long-term prognosis of stage II/III GC patients with postoperative complications, and it was the first to report the prognostic effect of the TI on GC patients with postoperative complications. This study also provides a preliminary basis for future multicenter or prospective studies.

\section{Conclusion}

This study showed that PCs can significantly reduce the 5-year OS and RFS rates of patients with GC. Postoperative adjuvant chemotherapy can offset the negative impacts of PCs on the long-term survival of patients with stage III GC; for stage II patients, the above offset effect is affected by the TI, and AC may not improve the survival of stage II patients with PCs in whom postoperative adjuvant chemotherapy is initiated $\geq 6$ weeks. However, the findings of this study still need to be further verified via external data, especially Western data. 


\section{Declarations}

\section{Acknowledgments}

\section{Human rights statement and informed consent}

All procedures followed were in accordance with the ethical standards of the responsible committee on human experimentation (institutional and national) and with the Helsinki Declaration of 1964 and later versions. Informed consent or substitute for it was obtained from all patients for being included in the study.

\section{Consent for publication}

This article doesn't report an individual participant's data in any form.

\section{Availability of data and materials}

The datasets used and/or analyzed during the current study are available from the corresponding author on reasonable request.

\section{Author Contributions:}

Drs Shen LL and Lu J contributed equally to this work and should be considered first coauthors.

Drs Zheng $\mathrm{CH}$ and Huang $\mathrm{CM}$ had full access to all of the data in the study and take responsibility for the integrity of the data and the accuracy of the data analysis. Concept and design: Shen LL, Lu J , Zheng CH Acquisition, analysis, or interpretation of data: Lu J, Shen LL, Xue Z , Xu BB, Wu D

Drafting of the manuscript: Shen LL,Lu J, Zheng CH, Huang CM.

Critical revision of the manuscript for important intellectual content: Lu J, Zheng CH, Shen LL, Xie JW, Zheng $\mathrm{HL}$, Zheng $\mathrm{CH}$, Huang $\mathrm{CM}$.

Statistical analysis: Shen LL,Lu J, Zheng HL, Zheng CH, Li P

Obtained funding: Lu J, Zheng CH, Xie JW, Li P

Administrative, technical, or material support: Huang CM.

Supervision: Huang CM.

Conflict of Interest Disclosures: None reported.

Disclosure: There are no conflicts of interest or financial ties to disclose from any author. 


\section{References}

1. Bray F, Ferlay J, Soerjomataram I, et al. Global cancer statis-tics 2018: GLOBOCAN estimates of incidence and mortality worldwide for 36 cancers in 185 countries. CA Cancer J Clin. 2018; 68(6):394-424.

2. Korean practice guideline for gastric cancer 2018: an evidence-based, multi-disciplinary approach. Journal of gastric cancer 2019; 19(1):1-48.

3. Sano T, Sasako M, Yamamoto S, et al. Gastric cancer surgery: morbidity and mortality results from a prospective randomized controlled trial comparing D2 and extended para-aortic lymphadenectomyJapan Clinical Oncology Group study 9501. J Clin Oncol. 2004; 22(14):2767-2773.

4. Bonenkamp JJ, Songun I, Hermans J, et al. Randomised comparison of morbidity after D1 and D2 dissection for gastric cancer in 996 Dutch patients. Lancet. 1995; 345(8952):745-748.

5. Cuschieri A, Fayers P, Fielding J, et al. Postoperative morbidity and mortality after D1 and D2 resections for gastric cancer: pre-liminary results of the MRC randomised controlled surgical trial. The Surgical Cooperative Group Lancet. 1996; 347(9007):995-999.

6. Walker KG, Bell SW, Rickard MJ, et al. Anastomotic leakage is predictive of diminished survival after potentially curative resection for colorectal cancer. Ann Surg. 2004; 240(2):255-259.

7. Artinyan A, Orcutt ST, Anaya DA, et al. Infectious postoperative complications decrease long-term survival in patients undergoing curative surgery for colorectal cancer: a study of 12,075 patients. Ann Surg. 2015; 261(3):497-505.

8. Lerut T, Moons J, Coosemans $\mathrm{W}$, et al. Postoperative complications after transthoracic esophagectomy for cancer of the esophagus and gastroesophageal junction are correlated with early cancer recurrence: role of systematic grading of complications using the modified Clavien classification. Ann Surg. 2009; 250(5):798-807.

9. Rizk NP, Bach PB, Schrag D, et al. The imACt of complications on outcomes after resection for esophageal and gastroesophageal junction carcinoma. J Am Coll Surg. 2004; 198(1):42-50.

10. Sierzega M, Kolodziejczyk P, Kulig J. ImACt of anastomotic leakage on long-term survival after total gastrectomy for carcinoma of the stomach. Br J Surg. 2010; 97(7):1035-1042.

11. Tokunaga $M$, Tanizawa $Y$, Bando $E$, et al. Poor survival rate in patients with postoperative intraabdominal infectious complications following curative gastrectomy for gastric cancer. Ann Surg Oncol. 2013; 20(5):1575-1583.

12. Kubota T, Hiki N, Sano T, et al. Prognostic significance of complications after curative surgery for gastric cancer. Ann Surg Oncol. 2014; 21(3):891-898.

13. Bohle $B$, Pera $M$, Pascual $M$, et al. Postoperative intra-abdominal infection increases angiogenesis and tumor recurrence after surgical excision of colon cancer in mice. Surgery. 2010;147 (1):120-126.

14. Yoshida K, Kodera Y, Kochi M, et al. Addition of docetaxel to oral fluoropyrimidine improves efficacy in patients with stage III gastric cancer: Interim analysis of JACCRO GC- 07, a randomized controlled trial. J Clin Oncol. 2019; 37(15):1296-1304. 
15. Biagi JJ, Raphael MJ, Mackillop WJ, et al. Association between time to initiation of adjuvant chemotherapy and survival in colorectal cancer: a systematic review and meta-analysis. JAMA 2011; 305(22):2335-2342.

16. Nachiappan S, Askari A, Mamidanna R, et al. The imACt of adjuvant chemotherapy timing on overall survival following colorectal cancer resection. Eur J Surg Oncol 2015; 41(12):1636-1644.

17. Tsujimoto $\mathrm{H}$, Ichikura $\mathrm{T}$, Ono $\mathrm{S}$, et al. ImACt of postoperative infection on long-term survival after potentially curative resection for gastric cancer. Ann Surg Oncol. 2009; 16(2):311-318.

18. Clavien PA, Barkun J, de Oliveira ML, et al. The Clavien-Dindo classification of surgical complications: five-year experience. Ann Surg. 2009; 250(2):187-196.

19. Fujiya $\mathrm{K}$, Tokunaga $\mathrm{M}$, Mori $\mathrm{K}$, et al. Long-term survival in patients with postoperative intra-abdominal infectious complications after curative gastrectomy for gastric cancer: A propensity score matching analysis. Ann Surg Oncol. 2016;23 (Suppl 5):809-816.

20. Sierzega M, Kolodziejczyk P, Kulig J, et al. Polish Gastric Cancer Study Group. ImACt of anastomotic leakage on long-term survival after total gastrectomy for carcinoma of the stomach. Br J Surg. 2010; 97(7):1035-1042.

21. Kun-Kun Li, Yin-Jian Wang, Xue-Hai Liu, et al. The effect of postoperative complications on survival of patients after minimally invasive esophagectomy for esophageal cancer. Surg. Endosc. 2017; 31(9):3475-3482.

22. Bin-bin Xu, Jun Lu, Zhi-fang Zheng, et al. The predictive value of the preoperative C-reactive proteinalbumin ratio for early recurrence and chemotherapy benefit in patients with gastric cancer after radical gastrectomy: using randomized phase III trial data. 2019;22(5):1016-1028.

23. Z. Li, B. Bai, Y. Zhao, et al. Severity of complications and long-term survival after laparoscopic total gastrectomy with $\mathrm{D} 2$ lymph node dissection for advanced gastric cancer: A propensity scorematched, case-control study. Int. J. Surg. 2018;54(Pt A):62-69.

24. Nan Jiang, Jing-Yu Deng, Xue-Wei Ding, et al. Effect of complication grade on survival following curative gastrectomy for carcinoma., World J. Gastroenterol. 2014; 20(25):8244-8252.

25. J. H. Saunders, F. Yanni, M. S. Dorrington, et al. ImACt of postoperative complications on disease recurrence and long-term survival following of esophagogastric cancer resection. $\mathrm{Br} \mathrm{J}$ Surg. 2020;107(1):103-112.

26. Masanori Tokunaga, Yukinori Kurokawa, Ryunosuke Machida, et al. ImACt of postoperative complications on survival outcomes in patients with gastric cancer: exploratory analysis of a randomized controlled JCOG1001 trial. Gastric Cancer 2020;24 (1):214-223.

27. Mirnezami A, Mirnezami R, Chandrakumaran K, et al. Increased local recurrence and reduced survival from colorectal cancer following anastomotic leak: systematic review and meta-analysis. Ann Surg 2011; 253(5):890-899.

28. Katai H, Mizusawa J, Katayama $\mathrm{H}$, et al. Short-term surgical out-comes from a phase III study of laparoscopy-assisted versus open distal gastrectomy with nodal dissection for clinical stage IA/IB 
gastric cancer: Japan Clinical Oncology Group Study JCOG0912. Gastric Cancer. 2017; 20(4):699708.

29. Okholm C, Goetze JP, Svendsen LB, et al. Inflammatory response in laparoscopic vs. open surgery for gastric cancer. Scand J Gastroenterol. 2014; 49(9):1027-34.

30. Sergei I Grivennikov, Florian R Greten, Michael Karin, et al. Immunity, inflammation, and cancer. Cell. 2010;140(6):883-899.

31. Mantovani A, Allavena P, Sica A, et al. Cancer-related inflammation. Nature. 2008;454(7203):436-444.

32. Liao WC, Lin JT, Wu CY, et al. Serum interleukin-6 level but not genotype predicts survival after resection in stages II and III gastric carcinoma. Clin Cancer Res. 2008; 14(2):428-434

33. Jacqueline Bromberg, Timothy C Wang. IL-6 and STA T3 complete the link. Cancer Cell. 2009; 15(2) : 79-80.

34. Zyao G, Zhu G, Huang Y, et al. IL-6 mediates the signal pathway of JAK-STAT3- VEGF-C promoting growth, invasion and lymphangiogenesis in gastric cancer. Oncol Rep. 2016;35(3):1787-1795.

35. Wu Y, Deng J, Rychahou PG, et al. Stabilization of SNAIL by NF-kappaB is required for inflammationinduced cell migration and invasion. Cancer Cell. 2009; 15(5):416-428.

36. Yamasaki M, Nakajima K, Takiguchi S, et al. Which is a more reliable indicator of survival after gastric cancer surgery: Postoperative complication occurrence or C-reactive protein elevation? J Surg Oncol. 2015; 112(8):894-899.

37. Bang YJ, Kim YW, Y ang HK, et al. Adjuvant capecitabine and oxaliplatin for gastric cancer after D2 gastrectomy (CLASSIC): a phase 3 open-label, randomised controlled trial. Lancet. 2012; 379(9813):315-321.

38. Sakuramoto $S$, Sasako $M, Y$ amaguchi T, et al. Adjuvant chemotherapy for gastric cancer with $S-1$, an oral fluoropyrimidine. N Engl J Med. 2007; 357(18):1810-1820.

39. Sasako M, Sakuramoto $S$, Katai $H$, et al. Five-year outcomes of a randomized phase III trial comparing adjuvant chemotherapy with S-1 versus surgery alone in stage II or III gastric cancer. J Clin Oncol. 2011; 29(33):4387-4393.

40. Selena S. Li, BS, Brooks V. Udelsman, et al. ImACt of Postoperative Complication and Completion of Multimodality Therapy on Survival in Patients Undergoing Gastrectomy for Advanced Gastric Cancer. J Am Coll Surg. 2020; 230(6):912-924.

41. N. Jiang, J.-Y. Deng, X.-W. Ding, et al. Effect of complication grade on survival following curative gastrectomy for carcinoma. World J. Gastroenterol. 2014; 20(25):8244-8252.

42. Z. Li, B. Bai, Y. Zhao, et al. Severity of complications and long-term survival after laparoscopic total gastrectomy with $\mathrm{D} 2$ lymph node dissection for advanced gastric cancer: A propensity scorematched, case-control study. Int. J. Surg. 2018; 54(Pt A):62-69.

43. C. S. Arhi, E. M. Burns, G. Bouras, et al. Complications after discharge and delays in adjuvant chemotherapy following colonic resection: a cohort study of linked primary and secondary care data. Colorectal Dis. 2019; 21(3):307-314. 
44. Xu F, Rimm AA, Fu P, et al. The imACt of delayed chemotherapy on its completion and survival outcomes in stage II colon cancer patients. PLoS ONE. 2014; 9 (9): e107993.

45. K.-K. Li, Y .-J. Wang, X.-H. Liu, et al. The effect of postoperative complications on survival of patients after minimally invasive esophagectomy for esophageal cancer. Surg. Endosc. 2017; 31(9):34753482.

46. Biagi JJ, Raphael MJ, Mackillop WJ, et al. Association between time to initiation of adjuvant chemotherapy and survival in colorectal cancer: a systematic review and meta-analysis. JAMA. 2011; 305(22):2335-2342.

47. Nachiappan S, Askari A, Mamidanna R, et al. The imACt of adjuvant chemotherapy timing on overall survival following colorectal cancer resection. Eur J Surg Oncol. 2015; 41(12):1636-1644.

\section{Figures}

Fig. 1

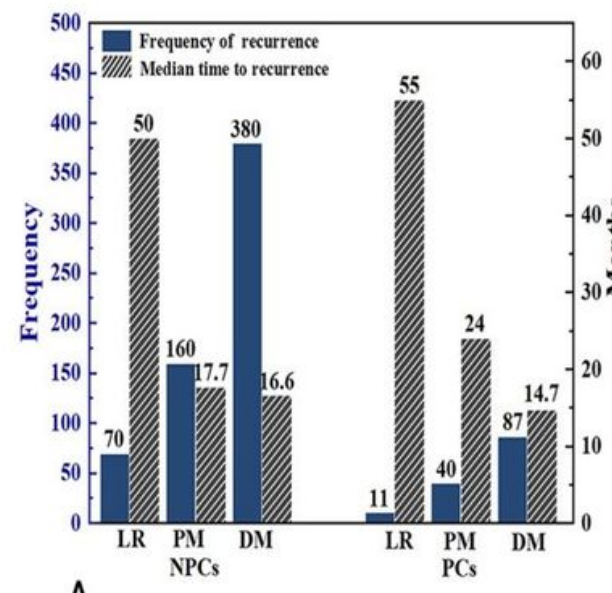

A

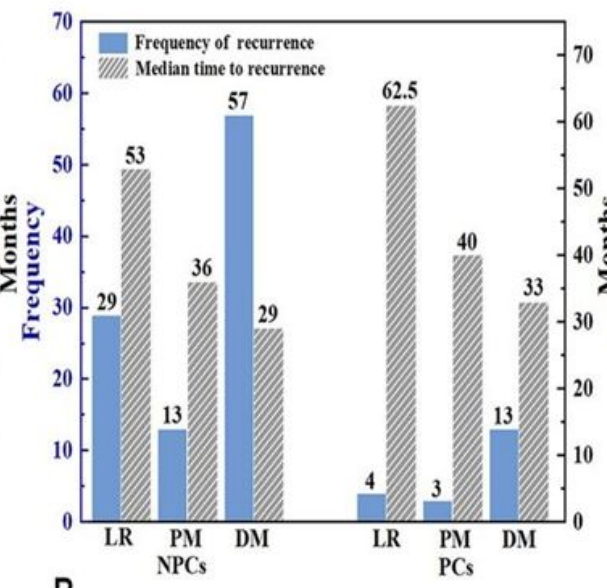

B

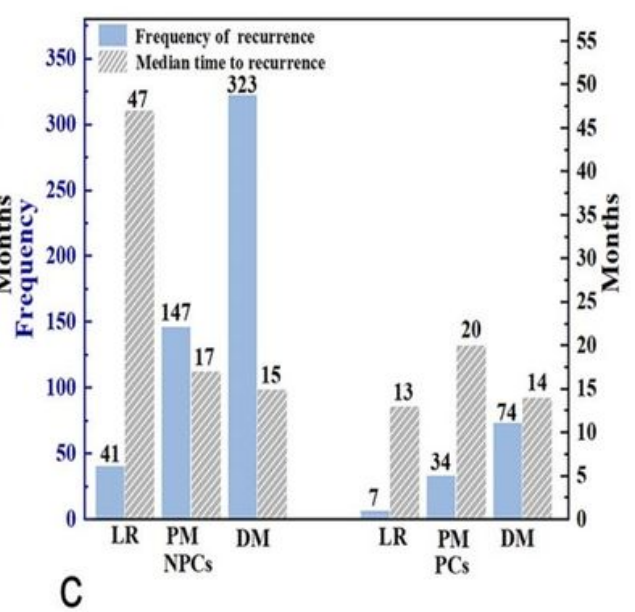

\section{Figure 1}

Frequency distribution of Anastomotic site of recurrence and median recurrence time distribution in

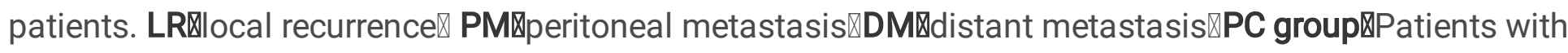
grade II and above complications ( $\geq$ grade II Clavien-Dindo) $\triangle N P C$ groupखPatients without complications

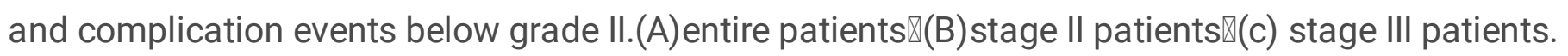


Fig. 2
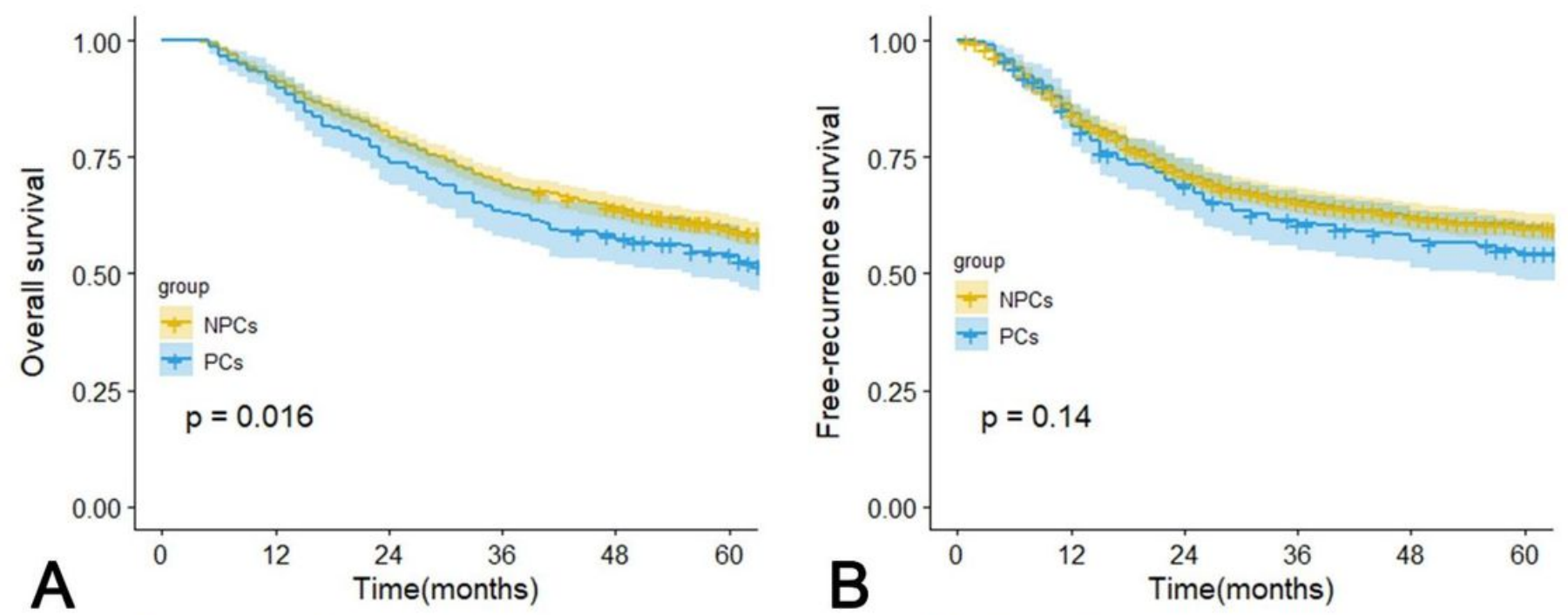

Number at risk

$\begin{array}{cccccc}\text { NPCs } 1295 & 1192 & 1041 & 907 & 829 & 668 \\ \text { PCs } 268 & 244 & 200 & 170 & 154 & 133\end{array}$

Number at risk

$\begin{array}{cccccc}\text { NPCS } 1295 & 1063 & 830 & 713 & 648 & 582 \\ \text { PCs } 268 & 216 & 172 & 150 & 135 & 121\end{array}$

\section{Figure 2}

Kaplan-Meier analyses of patients. (A)OS of PC group is lower(PC group $n=268$; NPC group $n=1295$, $p=0.016)$ and $(B)$ no significant difference was observed with respect to RFS in patients when they were $P C$ group or not $(p=0.14)$. 
Fig. 3

\section{Based on pathological staging set}

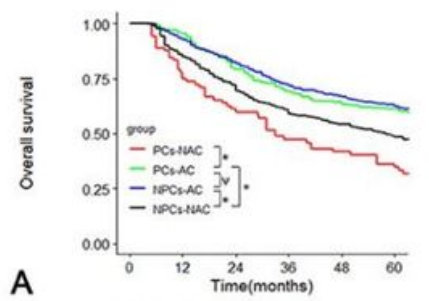

Number at risk

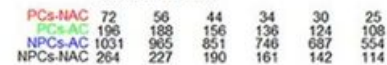

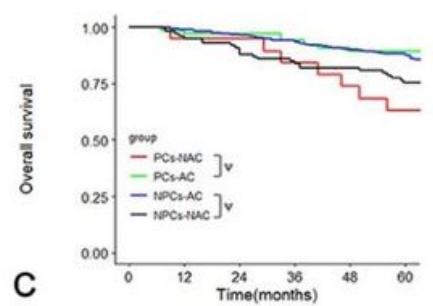

C

$$
\text { Number at risk }
$$

$\begin{array}{ccccccc}\text { PCSNACC } & 19 & 18 & 18 & 16 & 14 & 12 \\ \text { NPCSAC } 66 & 65 & 64 & 62 & 60 & 59 \\ \text { NPCSNACC } & 92 & 328 & 321 & 312 & 296 & 249 \\ \text { M. } & 95 & 90 & 81 & 81 & 67\end{array}$

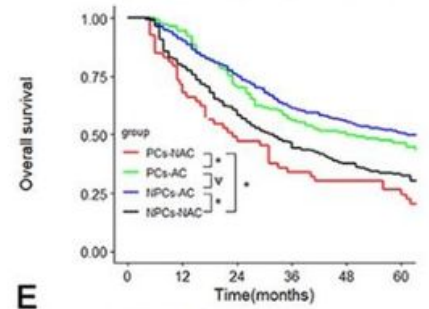

E

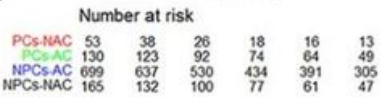

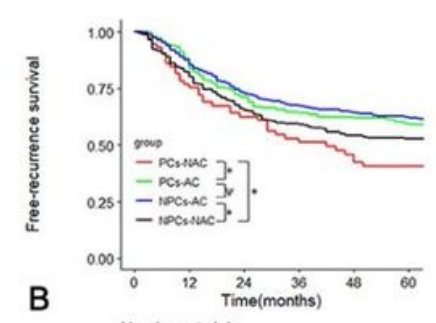

B
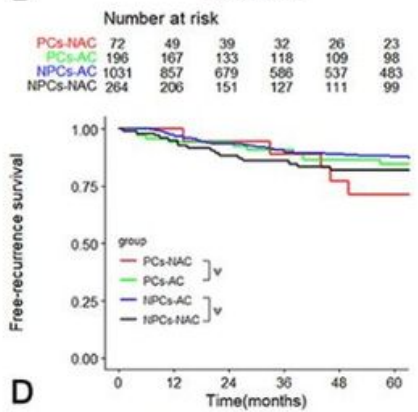

Number at risk
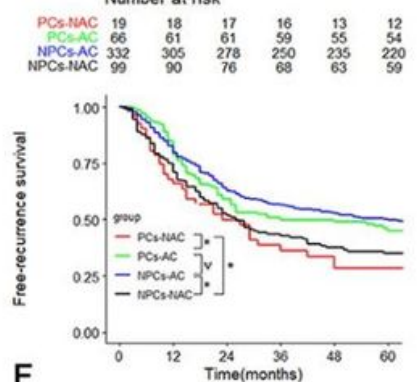

$\mathrm{F}$

Number at risk

PCS.MAC $53 \quad \begin{array}{lllll}51 & 22 & 16 & 13 & 11\end{array}$
Based on time interval ( $\mathrm{TI})$ from radical operation to initiation of adjuvant chemotherapy set
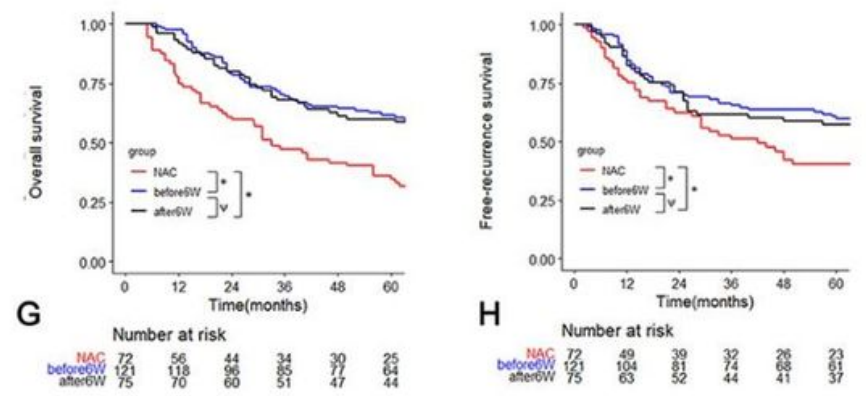

$\mathrm{H}$
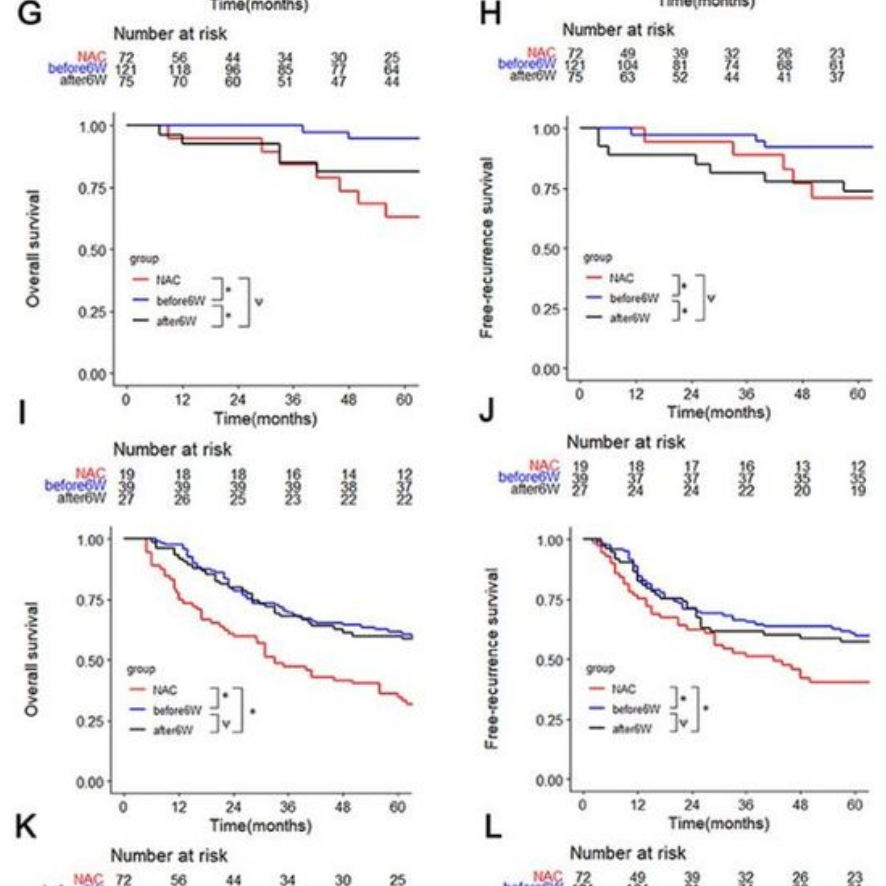

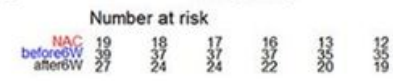

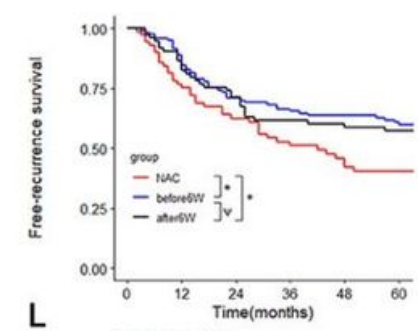

L Number at risk

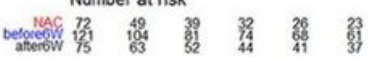

\section{Figure 3}

Kaplan-Meier analyses of survival, left panel based on pathological staging and right panel based on time interval ( $\mathrm{TI})$ from radical operation to initiation of adjuvant chemotherapy, cut-off value of 6 weeks for TI was used as in Figure 3.

left panel: Overall survival: (A) PC group could significantly benefit from AC(PCs-AC, $n=196$; PCs-NAC, $\mathrm{N}=72$; OS, $\mathrm{p} \otimes 0.002)$ \no significant difference was observed with respect to OS in PC group $(n=196)$ and NPC group $(n=798)$ when they were given AC (OS, pख0.440). (C) In subset of pStage II, no significant difference was observed with respect to OS in PC group (pStage II,PCs-AC: $n=66$; PCs-NAC: $n=19$ ) when they were given $A C$ or not (OS, p>0.05), (E) In subset of pStage III (pStage III, PCs-AC: $n=130$; PCs-NAC: $n=53)$ could significantly benefit from AC (OS, $p \otimes 0.000)$. Similar trends were seen on benefit of RFS in

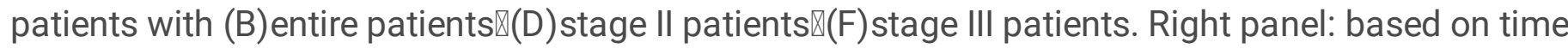
interval ( $\mathrm{TI}$ ) of initiation of adjuvant chemotherapy (G) PC group significantly benefit from AC when TI 6 weeks or $\mathrm{TI} \geq 6$ weeks (OS, $\mathrm{p}=0.000$ ), no significant difference was observed with respect to OS in patients

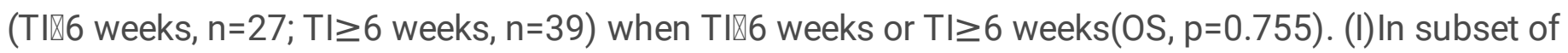


pStage II, compared with NAC( $n=19), P C$ group significantly benefit from AC when TI 86 weeks $(n=27,0 S$, $p=0.038)$ neither $T I \geq 6$ weeks $(n=39, O S, p=0.894)$. $(K)$ In subset of pStage III , compared with NAC $(n=53)$, PC group significantly benefit from AC either TI $₫ 6$ weeks $(n=48,0 S, p=0.005)$ or $T I \geq 6$ weeks $(n=82,0 S$, $p=0.011)$, no significant difference was observed with respect to OS in pStage III patients when TI 66 weeks or $\mathrm{Tl} \geq 6$ weeks(OS, $\mathrm{p}=0.843$ ). Similar trends were seen on benefit of RFS in patients with(H)entire

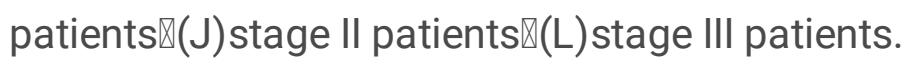

$(\delta: p<0.05 \llbracket \psi: p>0.05 \rrbracket P C s-N A C:$ PC group without adjuvant chemotherapy; PCs-AC: PC group with adjuvant chemotherapy; NPCs-AC: NAC group with adjuvant chemotherapy; NPCs-NAC: NAC group without adjuvant chemotherapy; NAC: never received postoperative adjuvant chemotherapy; TI $₫ 6$ weeks: initiation of postoperative adjuvant chemotherapy within 6 weeks; $T l \geq 6$ weeks: initiation of postoperative adjuvant chemotherapy after 6 weeks).

\section{Supplementary Files}

This is a list of supplementary files associated with this preprint. Click to download.

- Supplementalmaterial.docx 